\title{
Immunological response to quadrivalent HPV vaccine in treatment of recurrent respiratory papillomatosis
}

\author{
Robin E. A. Tjon Pian Gi ${ }^{1,5} \cdot$ Michel R. M. San Giorgi ${ }^{1,5} \cdot$ Michael Pawlita $^{2}$ • \\ Angelika Michel ${ }^{2}$ - Bettien M. van $\mathrm{Hemel}^{3}$ • Ed M. D. Schuuring ${ }^{3,5}$. \\ Edwin R. van den Heuvel ${ }^{4}$ - Bernard F. A. M. van der Laan ${ }^{1,5}$ - Frederik G. Dikkers ${ }^{1,5}$
}

Received: 30 September 2015/Accepted: 5 May 2016/Published online: 17 May 2016

(c) The Author(s) 2016. This article is published with open access at Springerlink.com

\begin{abstract}
Aim of this study was to explore influence of the quadrivalent HPV vaccine $\left(\right.$ Gardasil ${ }^{\circledR}$ ) on the immune status of recurrent respiratory papillomatosis (RRP) patients. In retrospective observational study, six RRP patients who received the quadrivalent HPV vaccine and whose HPV seroreactivity was measured were included. Multiplex HPV Serology was used to determine HPVspecific antibodies pre- and post-vaccination. Surgical interventions and patient records were analyzed. Five HPV6 and 1 HPV11 infected patient were included. Mean antibody reactivity against the associated HPV type rose from 1125 median fluorescence intensity (MFI) pre-vaccination to 4690 MFI post-vaccination $(p<0.001)$. Median post-vaccination follow-up was 4 years. Poisson regression analysis showed that the quadrivalent HPV
\end{abstract}

R. E. A. Tjon Pian Gi and M. R. M. San Giorgi contributed equally to this work.

Frederik G. Dikkers

f.g.dikkers@umcg.nl

1 Department of Otorhinolaryngology/Head and Neck Surgery, University of Groningen, University Medical Center Groningen, P.O. Box 30.001, 9700 RB Groningen, The Netherlands

2 Division of Molecular Diagnostics of Oncogenic Infections, German Cancer Research Center, Heidelberg, Germany

3 Department of Pathology, University of Groningen, University Medical Center Groningen, Groningen, The Netherlands

4 Department of Mathematics and Computer Science, Eindhoven University of Technology, Eindhoven, The Netherlands

5 Graduate School of Medical Sciences (Cancer Research Center Groningen), University of Groningen, Groningen, The Netherlands vaccine decreased the incidence rate of surgeries. The immune system of RRP patients is able to increase antibody reactivity against the associated HPV type. A double blind randomized controlled trial is needed to determine whether this immunological increase can cause decrease in number of surgeries.

Keywords Recurrent respiratory papillomatosis . Vaccine $\cdot$ Therapeutic drug · Immunology

\section{Introduction}

Infection with a subset of human papillomaviruses (HPV) can cause anogenital cancer, oropharyngeal cancer, condylomata acuminata and recurrent respiratory papillomatosis (RRP) [1, 2]. Since 2006 many national vaccination programs have started with the bivalent HPV vaccine $\left(\right.$ Cervarix ${ }^{\circledR}$, GlaxoSmithKline Biologicals s.a., Rixensart, Belgium) or the quadrivalent HPV vaccine Gardasil $^{\circledR}$, Merck \& co, Whitehouse Station, USA) targeting high-risk oncogenic HPV types 16 and 18. Papillomavirus vaccines are generally safe and highly effective [3]. The quadrivalent HPV vaccine is a subunit vaccine composed of the major capsid protein L1 primarily in the form of virus-like particles (VLPs) of low-risk HPV6 and 11 and high-risk HPV16 and 18. HPV6 and 11 cause $90 \%$ of genital warts [4]. It is expected that preventive global use of this HPV vaccine against cervical cancer will decrease the incidence of HPV6 and HPV11 related disease worldwide [5].

Recurrent respiratory papillomatosis (RRP) is a wartlike disease characterized by its unpredictable clinical course. It is associated with HPV6 and 11 for $80-100 \%$ of cases [6-10]. Therapy focuses on repeated surgical removal of exophytic lesions. Some patients may need over 
a 100 surgical interventions to keep the airway open and the voice sufficient [6].

Antibody response after vaccination with the quadrivalent HPV vaccine is higher than after natural infection in patients with high-risk HPV [3, 11]. Little is known about the antibody response for low-risk HPV. Increased seroreactivity after vaccination in RRP patients was only addressed in two case reports [12, 13]. After vaccination with the quadrivalent HPV vaccine, HPV seropositive women were protected against cervical and anogenital diseases from the corresponding HPV type [3]. Therefore, vaccination of RRP patients could be a potential treatment against HPV re-infection or auto-inoculation. In this independent exploratory study we investigated whether vaccination with the quadrivalent HPV vaccine results in increase of antibodies against the associated viruses.

\section{Materials and methods}

\section{Ethical considerations}

Patients included in this retrospective cohort study were clinically treated off-label with the quadrivalent HPV vaccine; there was no scientific intent. Due to great international interest in the use of this therapy, it was decided to publish these valuable data. Written approval of all patients was received.

Institutional Review Board approval for retrospective cohort research is not needed in The Netherlands. All patients approved use of information from their patient files, laboratory results and biopsy material, by signing a consent form.

Biopsy and resection material of the included patients were available in archives of our Department of Pathology. This study was performed according to the Code of Conduct for Proper Secondary Use of Human Tissue in The Netherlands, as well as to the applicable institutional and national guidelines [14].

\section{Patients}

Patients' charts and surgical reports of all RRP patients treated at the Department of Otorhinolaryngology/Head and Neck Surgery of the tertiary referral hospital University Medical Center Groningen, University of Groningen, The Netherlands were retrospectively analyzed. Inclusion criteria for this study were: (1) histological confirmation of RRP by an experienced head and neck pathologist, (2) the patient received the quadrivalent HPV vaccine with therapeutic intent, (3) HPV seroreactivity known pre- and post-vaccination.

Patient charts were reviewed on date of birth, gender, date of diagnosis, risk factors (gastroesophageal reflux disease (GERD) and asthma), follow-up, number of surgeries, complications of administration of the quadrivalent HPV vaccine and complications associated with RRP (carcinoma, tracheotomy). Patients with an age of onset younger than 18 years of age have juvenile onset RRP (JoRRP). Patients older than 18 years at onset of disease have adult onset RRP (AoRRP).

\section{Vaccination}

The quadrivalent HPV vaccine was clinically administered to RRP patients between March 2011 and January 2013. Vaccination was injected intramuscularly by normal dosage of VLP6 $20 \mu \mathrm{g}$, VLP11 $40 \mu \mathrm{g}$, VLP16 $40 \mu \mathrm{g}$, and VLP18 $20 \mu \mathrm{g}$ per injection $(0.5 \mathrm{ml})$. Injections were given following the same schedule as in preventive vaccination: at 0,2 and 6 months [15]. The time after the first administration was considered as 'post-vaccination'. The second and third vaccinations were administered for durability of the effect [15].

\section{Time frame}

A blood sample was taken immediately before the first injection of the quadrivalent HPV vaccine (pre-vaccination seroreactivity). A second blood sample was taken immediately before the third vaccination (representing postvaccination seroreactivity).

\section{Patient material}

\section{HPV type specific polymerase chain reaction $(P C R)$}

For each patient a stored paraffin block from the first formalin-fixed biopsy was selected, in which papilloma was histopathologically confirmed. To confirm presence of papilloma an experienced pathologist revised all biopsies. When quality or quantity of the first biopsy was not sufficient for PCR, the next sufficient biopsy was used. HPV typing was performed using the HPV consensus primer set GP5+/6+ with subsequent nucleotide sequence analysis. Details of this technique have been described before [6].

\section{Antibody seroreactivity}

Seroreactivity to the HPV major capsid L1 protein for both HPV6 and HPV11 was measured to monitor antibody response against the quadrivalent HPV vaccine. Blood samples were analyzed by the multiplex human papillomavirus serology, based on in situ-purified glutathione S-transferase proteins, as described by Waterboer et al. [16, 17]. Briefly, full-length L1 proteins were bacterially expressed as fusion proteins with $\mathrm{N}$ terminal glutathione-S- 
transferase (GST) and a C terminal tagging peptide (tag) and were affinity-purified in situ from cleared bacterial lysates through binding to glutathione casein-coated fluorescencelabeled polystyrene beads. A fusion protein consisting of GST and tag (GST-tag) without intervening viral antigen served for background determination. Each fusion protein was bound to a spectrally distinct bead set. Fusion proteinloaded bead sets were mixed. Sera were pre-incubated at 1:50 dilution in PBS containing $1 \mathrm{mg} / \mathrm{mL}$ casein, $2 \mathrm{mg} / \mathrm{mL}$ lysate from bacteria expressing GST-tag alone to block antibodies directed against residual bacterial proteins and GST-tag, $0.5 \%$ polyvinylalcohol (PVA, Sigma-Aldrich Chemie Gmbh Munich, Germany), $0.8 \%$ polyvinylpyrrolidone (PVP, Sigma-Aldrich Chemie Gmbh Munich, Germany) and $2.5 \%$ Superchemiblock (Millipore, Billerica, MA, USA) to suppress unspecific binding of antibodies to the beads themselves [17]. Serum dilutions were incubated with the same volume of mixed bead sets, resulting in a final serum dilution of 1:100. Bound antibodies were detected with biotinylated goat-antihuman $\operatorname{IgG}(\mathrm{H}+\mathrm{L})$ secondary antibody and streptavidin- $R$-phycoerythrin. A Luminex analyser (xMAP, Luminex Corp., Austin, TX, USA) was used to identify the internal color of the individual beads and to quantify their reporter fluorescence (expressed as median fluorescence intensity (MFI) of at least 100 beads per set per serum). Antibody reactivity, i.e. the amount of antigenspecific antibody bound per bead is expressed as net MFI values calculated as difference of MFI with HPV-protein minus MFI with GST-tag.

\section{Statistical analysis}

Analyses were performed using PASW statistics version 20.0 (SPSS Inc., Chicago, IL, USA) and SAS version 9.3 (SAS Institute, Inc., Cary, NC, USA).

Categorical variables are presented as number (percentage). Normally distributed variables are presented as mean \pm standard deviation. Data presented as $[x ; x]$ represents $95 \%$ confidence interval. $p$ value of $<0.05$ was considered statistically significant.

A paired $t$ test was used to analyze the difference between pre- and post MFI. Descriptive statistics were provided for the rate of surgical interventions (number of surgical interventions divided by time interval) before and after vaccinations. A Spearman correlation coefficient between the two rates was calculated. Poisson regression analysis (with a random intercept for subjects) was applied to investigate a possible effect of vaccine on the mean number of surgical interventions corrected for type of papilloma virus (HPV6 and HPV11) and age at onset. Subject's variable log time period was included in the regression analysis as offset parameter to adjust for different time intervals for subjects.
Since the analysis is only preliminary and exploratory, a sample size for a parallel group randomized clinical trial was calculated on the basis of an effect size that vaccination reduces the mean number of surgical interventions with $50 \%$. Formula four of Signorini et al. with a Bernoulli covariate was used [18].

\section{Results}

Nine RRP patients of the University Medical Center Groningen received the quadrivalent HPV vaccine. For six of them seroreactivity pre- and post-vaccination were known; these six patients were included in this exploratory study. Patients were diagnosed with RRP between 1981 and 2011, followed until August 1, 2015. Characteristics per patient are presented in Table 1. All included patients were male. The mean age of onset was 16 years (SD 16). Three patients (50\%) had JoRRP, three patients $(50 \%)$ had AoRRP. None of the patients had asthma or GERD. Five patients were infected with HPV6 and one patient was infected with HPV11.

The mean pre-vaccination antibody reactivity was 1125 MFI (SD 884). The mean post-vaccination antibody reactivity was 4690 MFI (SD 727). All individual antibody reactivities increased after vaccination, with a median rise of 3766 MFI (range 1199-4670). The mean MFI per patient rose significantly after vaccination $(p<0.001)$. The change of pre- and post-vaccination antibody reactivity of the associated viruses are represented in Fig. 1.

None of the patients experienced side effects or complications of the vaccination. The surgical course over time is presented in Fig. 2. The median pre-vaccination disease history was 3 years (range 1-30). The median post-vaccination follow-up was 4 years (range 3-4). The interval between surgeries ranged from 1 week to 7 years (Fig. 2). The average rates of surgical interventions for a period of a year were $4.34[1.11 ; 7.57]$ and $0.99[0.25 ; 1.73]$ before and after vaccination, respectively. Spearman correlation coefficient between the rates before and after was estimated at $-0.20(p=0.704)$.

Poisson regression analysis corrected for age at onset and type of HPV demonstrated a clinical effect of vaccination. The effect size was estimated at -1.20 [ -1.90 ; $-0.50]$. This meant that the mean number of surgical interventions in a specific time frame after vaccination decreased with approximately a factor of $3.3\left(=\exp ^{1.20}\right)$.

Based on the results of a simpler Poisson regression analysis (using only the vaccination variable and overdispersion), the sample size for detecting reduction in the mean number of surgical interventions after vaccination with a factor of 2 was calculated. If a theoretical trial period would be 1 year, the total number of patients in each 

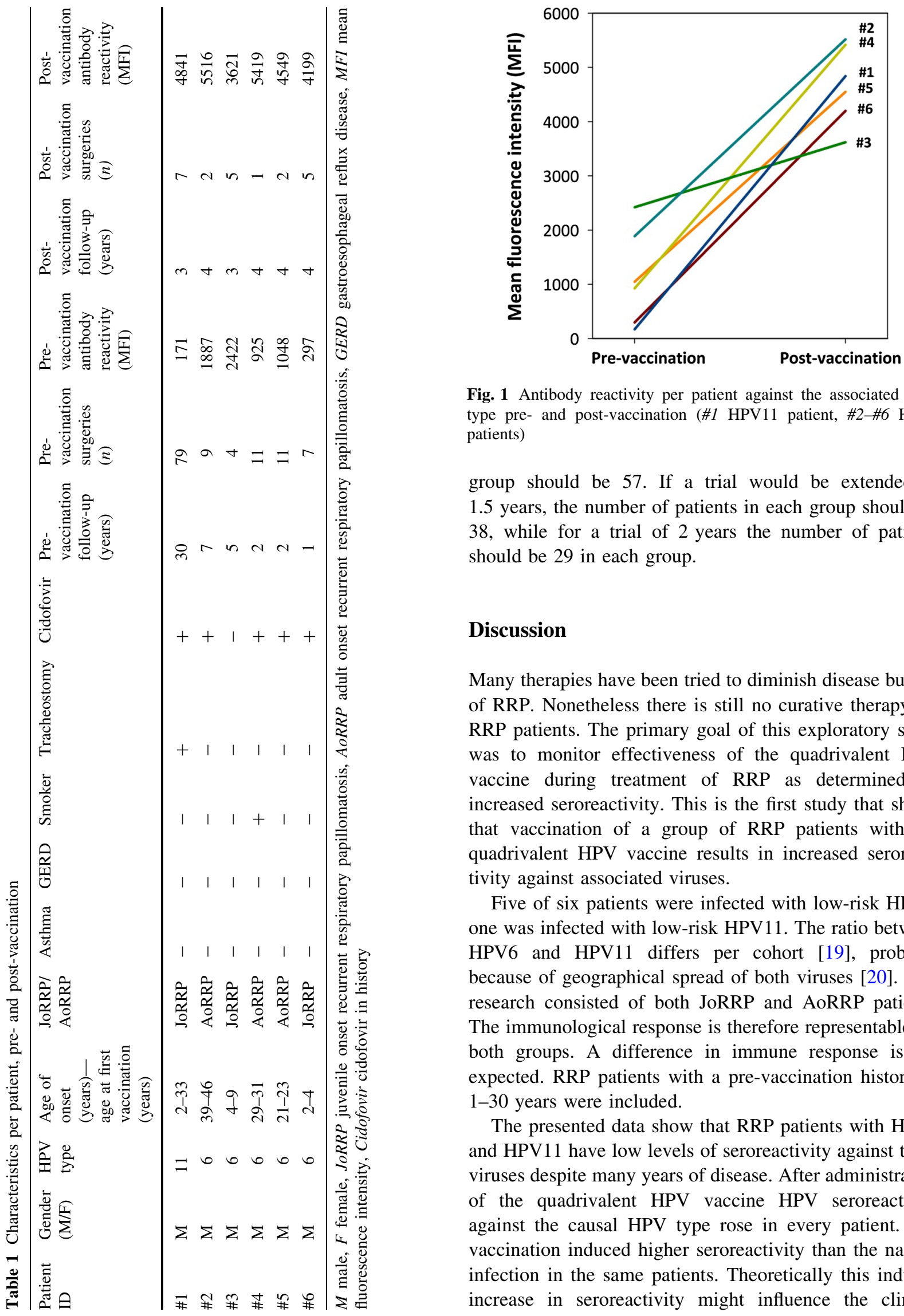

Fig. 1 Antibody reactivity per patient against the associated HPV type pre- and post-vaccination (\#1 HPV11 patient, \#2-\#6 HPV6 patients)

group should be 57. If a trial would be extended to 1.5 years, the number of patients in each group should be 38 , while for a trial of 2 years the number of patients should be 29 in each group.

\section{Discussion}

Many therapies have been tried to diminish disease burden of RRP. Nonetheless there is still no curative therapy for RRP patients. The primary goal of this exploratory study was to monitor effectiveness of the quadrivalent HPV vaccine during treatment of RRP as determined by increased seroreactivity. This is the first study that shows that vaccination of a group of RRP patients with the quadrivalent HPV vaccine results in increased seroreactivity against associated viruses.

Five of six patients were infected with low-risk HPV6, one was infected with low-risk HPV11. The ratio between HPV6 and HPV11 differs per cohort [19], probably because of geographical spread of both viruses [20]. This research consisted of both JoRRP and AoRRP patients. The immunological response is therefore representable for both groups. A difference in immune response is not expected. RRP patients with a pre-vaccination history of 1-30 years were included.

The presented data show that RRP patients with HPV6 and HPV11 have low levels of seroreactivity against these viruses despite many years of disease. After administration of the quadrivalent HPV vaccine HPV seroreactivity against the causal HPV type rose in every patient. The vaccination induced higher seroreactivity than the natural infection in the same patients. Theoretically this induced increase in seroreactivity might influence the clinical 
Fig. 2 Follow-up with all the patient $(n=6)$, pre- and were administered during the blue-marked period. \#1 HPV11 patient, \#2-\#6 HPV6 patients surgical interventions by age of post-vaccination. Vaccinations

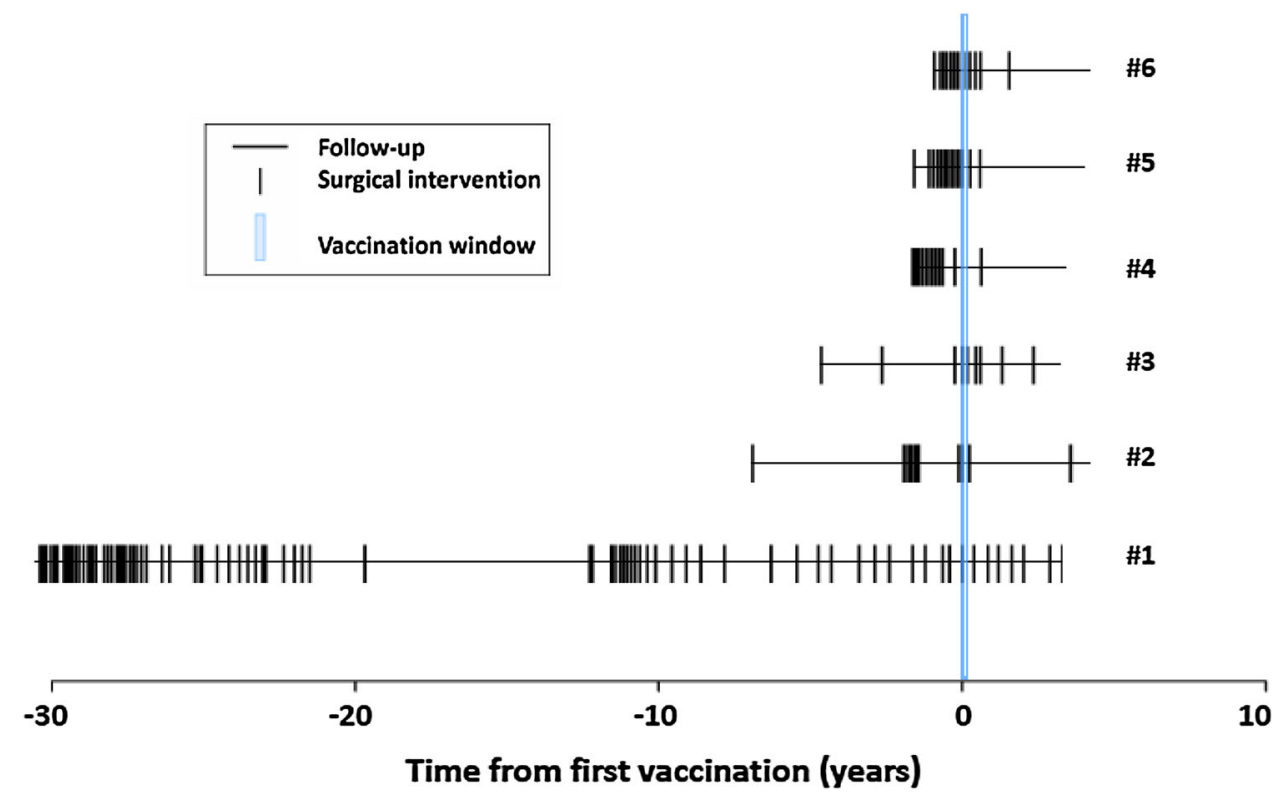

course of RRP by intensifying immune response and preventing re-infection.

This study is the first study to measure seroreactivity in a group of RRP patients. Results could be biased due to the small sample size and short follow-up. An effect of other adjuvant therapy on immunological response was not expected as patients did not receive any adjuvant therapy 1 month before, neither during or after vaccination. More research is needed to analyze the duration of the immunological response.

Chirila et al. concluded that the quadrivalent HPV vaccine was effective to diminish the recurrence rate of RRP in $85 \%$ of patients, although that study was retrospective and lacked a control group [21]. Furthermore the natural decreasing surgical rate of RRP was not taken into account $[6,22]$. It is unknown if there is a immune response after vaccination which explains a clinical response, therefore this study was conducted. The clinical response described in this article was only used for a power analysis for a future randomized controlled trial (RCT), as the sample size was too small to analyze the clinical course and to correct for the natural clinical course and other therapies (e.g. cidofovir). A RCT is needed to draw conclusions on the real clinical effect of the quadrivalent HPV vaccine. The proposed sample size for a trial with a followup of 2 years should be 29 patients per group.

\section{Conclusion}

RRP patients increase seroreactivity against the quadrivalent HPV vaccine, regardless of their age, age of onset, HPV type and severity of disease. Antibody reactivities to the associated viruses of all patients rose significantly. A double-blinded randomized controlled trial is needed to evaluate the effect of this vaccination on the clinical course. The quadrivalent HPV vaccine could be of future help in the treatment of RRP, as this research showed that vaccination causes a robust immunological response.

\section{Compliance with ethical standards}

Conflict of interest Prof. van den Heuvel is a consultant for MSD on the area of microbiological method validation.

Funding No specific funding.

Research involving human participants and/or animals: human patients involved in this retrospective cohort study, no animals. Institutional Review Board approval for retrospective cohort research is not needed in The Netherlands. All procedures performed in studies involving human participants were in accordance with the ethical standards of the institutional and/or national research committee and with the 1964 Helsinki Declaration and its later amendments or comparable ethical standards.

Informed consent Written approval of all patients was received.

Open Access This article is distributed under the terms of the Creative Commons Attribution 4.0 International License (http://crea tivecommons.org/licenses/by/4.0/), which permits unrestricted use, distribution, and reproduction in any medium, provided you give appropriate credit to the original author(s) and the source, provide a link to the Creative Commons license, and indicate if changes were made.

\section{References}

1. Chelimo C, Wouldes TA, Cameron LD, Elwood JM (2013) Risk factors for and prevention of human papillomaviruses (HPV), genital warts and cervical cancer. J Infect 66(3):207-217 
2. Gillison ML, Alemany L, Snijders PJ et al (2012) Human papillomavirus and diseases of the upper airway: head and neck cancer and respiratory papillomatosis. Vaccine 30(Suppl 5):F34F54

3. Olsson SE, Kjaer SK, Sigurdsson K et al (2009) Evaluation of quadrivalent HPV 6/11/16/18 vaccine efficacy against cervical and anogenital disease in subjects with serological evidence of prior vaccine type HPV infection. Hum Vaccin 5(10):696-704

4. Haupt RM, Sings HL (2011) The efficacy and safety of the quadrivalent human papillomavirus 6/11/16/18 vaccine gardasil. J Adolesc Health 49(5):467-475

5. Future II (2007) Study group. Quadrivalent vaccine against human papillomavirus to prevent high-grade cervical lesions. N Engl J Med 356(19):1915-1927

6. Tjon Pian Gi RE, San Giorgi MR, Slagter-Menkema L et al (2015) Clinical course of recurrent respiratory papillomatosis: comparison between aggressiveness of human papillomavirus- 6 and human papillomavirus-11. Head Neck 37(11):1625-1632

7. Sanchez GI, Jaramillo R, Cuello G et al (2013) Human papillomavirus genotype detection in recurrent respiratory papillomatosis (RRP) in colombia. Head Neck 35(2):229-234

8. Draganov P, Todorov S, Todorov I, Karchev T, Kalvatchev Z (2006) Identification of HPV DNA in patients with juvenile-onset recurrent respiratory papillomatosis using SYBR green real-time PCR. Int J Pediatr Otorhinolaryngol 70(3):469-473

9. Gerein V, Rastorguev E, Gerein J, Draf W, Schirren J (2005) Incidence, age at onset, and potential reasons of malignant transformation in recurrent respiratory papillomatosis patients: 20 years experience. Otolaryngol Head Neck Surg 132(3):392-394

10. Wiatrak BJ, Wiatrak DW, Broker TR, Lewis L (2004) Recurrent respiratory papillomatosis: a longitudinal study comparing severity associated with human papilloma viral types 6 and 11 and other risk factors in a large pediatric population. Laryngoscope 114(11 Pt 2 Suppl 104):1-23

11. Villa LL, Costa RL, Petta CA et al (2005) Prophylactic quadrivalent human papillomavirus (types $6,11,16$, and 18) L1 viruslike particle vaccine in young women: a randomised double-blind placebo-controlled multicentre phase II efficacy trial. Lancet Oncol 6(5):271-278

12. Forster G, Boltze C, Seidel J, Pawlita M, Muller A (2008) Juvenile laryngeal papillomatosis-immunisation with the polyvalent vaccine gardasil. Laryngorhinootologie 87(11):796-799

13. Pawlita M, Gissmann L (2009) Recurrent respiratory papillomatosis: indication for HPV vaccination? Dtsch Med Wochenschr 134(Suppl 2):S100-S102

14. Code for proper secondary use of human tissue in The Netherlands. Updated 2002. http://www.federa.org. Accessed 17 July 2014

15. Schiller JT, Castellsague X, Garland SM (2012) A review of clinical trials of human papillomavirus prophylactic vaccines. Vaccine 30(Suppl 5):F123-F138

16. Waterboer T, Sehr P, Michael KM et al (2005) Multiplex human papillomavirus serology based on in situ-purified glutathione s-transferase fusion proteins. Clin Chem 51(10):1845-1853

17. Waterboer T, Sehr P, Pawlita M (2006) Suppression of nonspecific binding in serological luminex assays. J Immunol Methods 309(1-2):200-204

18. Signorini DF (1991) Sample size for poisson regression. Biometrika 78(2):446-450

19. Donne AJ, Hampson L, Homer JJ, Hampson IN (2010) The role of HPV type in recurrent respiratory papillomatosis. Int J Pediatr Otorhinolaryngol 74(1):7-14

20. de Sanjose S, Diaz M, Castellsague X et al (2007) Worldwide prevalence and genotype distribution of cervical human papillomavirus DNA in women with normal cytology: a meta-analysis. Lancet Infect Dis 7(7):453-459

21. Chirila M, Bolboaca SD (2014) Clinical efficiency of quadrivalent HPV (types 6/11/16/18) vaccine in patients with recurrent respiratory papillomatosis. Eur Arch Otorhinolaryngol 271(5):1135-1142

22. San Giorgi MR, Tjon Pian Gi RE, Dikkers FG (2015) The clinical course of recurrent respiratory papillomatosis after the use of cidofovir is influenced by multiple factors. Eur Arch Otorhinolaryngol 272(7):1819-1820 